\title{
Enterospora sp., an intranuclear microsporidian infection of hermit crab Eupagurus bernhardus
}

\author{
G. D. Stentiford* ${ }^{*}$ K. S. Bateman \\ Centre for Environment, Fisheries and Aquaculture Science (CEFAS), Barrack Road, Weymouth, Dorset DT4 8UB, UK
}

\begin{abstract}
Recent work at our laboratory has led to the discovery of a new genus of microsporidian parasite residing in the family Enterocytozoonidae. The type species of this new genus, Enterospora canceri, is an intranuclear parasite infecting the hepatopancreatocytes of the decapod crustacean Cancer pagurus. Here we provide the second description of a parasite within the genus Enterospora, this time infecting the hermit crab Eupagurus bernhardus from UK waters. The pathological manifestation and ultrastructural features of the hermit crab parasite are very similar to those described for E. canceri. Further taxonomic comparisons based upon ultrastructural and molecular affinities of Enterospora are now required to define firmer links between this new genus within the Enterocytozoonidae and all other microsporidian families. The opportunistic nature of the discovery of a second intranuclear microsporidian within the Crustacea suggests that their presence may be more common than in higher animal groups.
\end{abstract}

KEY WORDS: Eupagurus bernhardus · Enterocytozoon · Enterocytozoonidae · Enterospora · Epithelium $\cdot$ Hermit crab $\cdot$ Hepatopancreas $\cdot$ Nucleospora $\cdot$ Intranuclear $\cdot$ Nucleus $\cdot$ Crustacea

\section{INTRODUCTION}

Until the discovery of Enterospora canceri in European edible crab Cancer pagurus by Stentiford et al. (2007, this issue), microsporidians with features of the family Enterocytozoonidae had only been described from 2 genera: one (Enterocytozoon) cytoplasmic within mammalian hosts, the other (Nucleospora) intranuclear within finfish hosts. While E. canceri possesses features of both existing genera and has developmental stages typical of the family, its invertebrate hosting coupled with its infection location and specific development stages suggested that Enterospora is a separate genus. As such, the discovery provides an interesting link between existing members of the family and their apparently disparate neighbour families within the Microsporidia. Here we describe a second example of Enterospora sp., this time infecting the hermit crab Pagurus bernhardus (Decapoda: Anomura) captured from the Irish Sea, United Kingdom. As such, it is a second case of an intranuclear microsporidian in the Crustacea. Whether the parasite from $E$. bernhardus is the same as that reported from European edible crab Cancer pagurus captured from the English Channel, or whether this marks the second species of the genus Enterospora remains to be shown.

\section{MATERIALS AND METHODS}

Thirty hermit crabs Eupagurus bernhardus were captured as a by-catch during the UK National Marine Monitoring Programme (NMMP) annual survey in June 2003 from a site in Liverpool Bay, Irish Sea $\left(53^{\circ} 28.66^{\prime} \mathrm{N}, 3^{\circ} 41.79^{\prime} \mathrm{W}\right)$. Hermit crabs were placed into running seawater for up to $1 \mathrm{~h}$ before preparation for histology and electron microscopy. Crabs were anaesthetized by chilling on ice for $30 \mathrm{~min}$ before removal of heart, gonad, gill, muscle and hepatopancreas. Dissected organs were fixed for $24 \mathrm{~h}$ in $10 \%$ Davidson's seawater fixative (Hopwood 1996) before transfer 
to $70 \%$ industrial methylated spirit (IMS). Fixed samples were processed to wax in a vacuum infiltration processor using standard protocols. Sections were cut at 3 to $5 \mu \mathrm{m}$ on a rotary microtome and resulting tissue sections were mounted onto glass slides before staining with haematoxylin and eosin (H\&E). Stained sections were analysed by light microscopy (Eclipse E800, Nikon) and digital images were obtained using the Lucia $^{\mathrm{TM}}$ Screen Measurement System (Nikon). Hepatopancreas samples prepared for histology were also preserved for electron microscopy. Small blocks of tissue $\left(2 \mathrm{~mm}^{3}\right)$ were fixed in $2.5 \%$ glutaraldehyde in $0.1 \mathrm{M}$ sodium cacodylate buffer $(\mathrm{pH} 7.4)$ and $1.75 \%$ sodium chloride for $2 \mathrm{~h}$ at room temperature. Fixed tissue samples were rinsed in $0.1 \mathrm{M}$ sodium cacodylate buffer with $1.75 \%$ sodium chloride ( $\mathrm{pH} 7.4)$ and postfixed for $1 \mathrm{~h}$ at $4^{\circ} \mathrm{C}$ in $1 \%$ osmium tetroxide, reduced with $1.75 \%$ potassium ferrocyanide in $0.1 \mathrm{M}$ sodium cacodylate buffer. Specimens were washed in 3 changes of $0.1 \mathrm{M}$ sodium cacodylate buffer and stained en bloc in $0.5 \%$ aqueous uranyl acetate for $1 \mathrm{~h}$. Specimens were embedded in epoxy resin 812 (Agar Scientificpre-mix kit 812) following dehydration through a graded acetone series. Thick sections were stained with Toluidine Blue for viewing with a light microscope to identify suitable target areas. Ultra thin sections (70 to $90 \mathrm{~nm}$ ) of these areas, mounted on uncoated copper grids and stained with uranyl acetate and Reynolds' lead citrate (Reynolds 1963), were examined using a JEOL 1210 transmission electron microscope. Dimensions were assessed using the on-board measurement systems associated with this microscope.

\section{RESULTS}

Hermit crabs displayed no external signs of disease. One specimen from the 30 assessed showed a severe degeneration of the hepatopancreas typical for that described previously in Cancer pagurus infected with the intranuclear microsporidian Enterospora canceri (Stentiford et al. 2007). The normal tubular structure of the hepatopancreas was almost entirely destroyed, with detachment of epithelial cells from the tubule basement membrane, a liberation of presumably free parasite spores to the tubule lumen and a pronounced haemocytic encapsulation response to affected tubules. Although the disease state was advanced, it was still possible to appreciate the intranuclear nature of the infection since hypertrophic, eosinophilic and granular nuclei were frequent amongst liberated spores and cellular debris within the bounds of the existing tubule basement membrane. In addition to the principle infection, colonies of Gram-negative bacteria were also apparent within the remnant tubules (Fig. 1).
Different life cycle stages of a microsporidian parasite were observed within the affected nuclei of epithelial cells of the hepatopancreatic tubules. In this example of presumably advanced infection, all tubular epithelial cells and their nuclei were implicated. Uninucleate meronts were not detected within infected nuclei, but presumptive subsequent stages were observed as plasmodia containing between 4 and $>20$ parasite nuclei in section. Larger plasmodia appeared to be lobed and occupied the majority of the hypertrophic host nucleus, with margination of the host chromatin. Typical of the genus Enterospora, early stage plasmodia contained a granular cytoplasm, were contained by a simple electron-dense membrane, developed small spherical membrane-bound vesicles and later, larger electron-lucent vacuoles. At this stage, paired parasite nuclei were closely opposed (Fig. 2A-D). Next, multiple copies of apparatus resembling the polar filament and anchoring disk of mature microsporidian spores formed within the plasmodial cytoplasm and became closely associated with the large electron-lucent vacuoles (Fig. 2E,F). Although sporoblasts were not observed budding from the sporogonal plasmodia, liberated sporoblasts were observed in direct contact with the host nucleoplasm. At this stage, the early polaroplast and developing polar filament and anchoring disks were present within a relatively electron-lucent and granular sporoblast cytoplasm. Occasionally small bleb-like protrusions emerged from the sporoblast outer membrane (Fig. 3A). Sporoblast stages matured to electron-dense spore stages that also were not contained within any sporophorous vesicle but, instead, lay in direct contact with the host nucleoplasm (Fig. 3B). In many cases, large numbers of spores (several tens to $>100$ ) were observed within a given section of host nucleus, suggesting that at least several hundred can be present within the nucleus as a whole (Fig. 3C). Spores contained 4 to 5 turns of the polar filament and measured $1.4 \pm 19.7 \times$ $0.7 \pm 11.5 \mu \mathrm{m}$ (mean $\mathrm{n}=30$ ) and were contained within a trilaminate wall consisting of a cell membrane $(7 \pm 0.4 \mathrm{~nm})$, endospore $(30 \pm 0.9 \mathrm{~nm})$ and exospore $(15 \pm 1.4 \mathrm{~nm})$. Gram-negative bacterial colonies were observed amongst cellular debris and remnants of the degenerated tubules (Fig. 3D, Gram stain not shown). Infected host nuclei frequently contained several development stages of the microsporidian, suggesting asynchrony between infections, even within the same host cell and nucleus (Fig. 4).

\section{DISCUSSION}

The parasite described herein is the second reported example of an intranuclear microsporidian in an inver- 

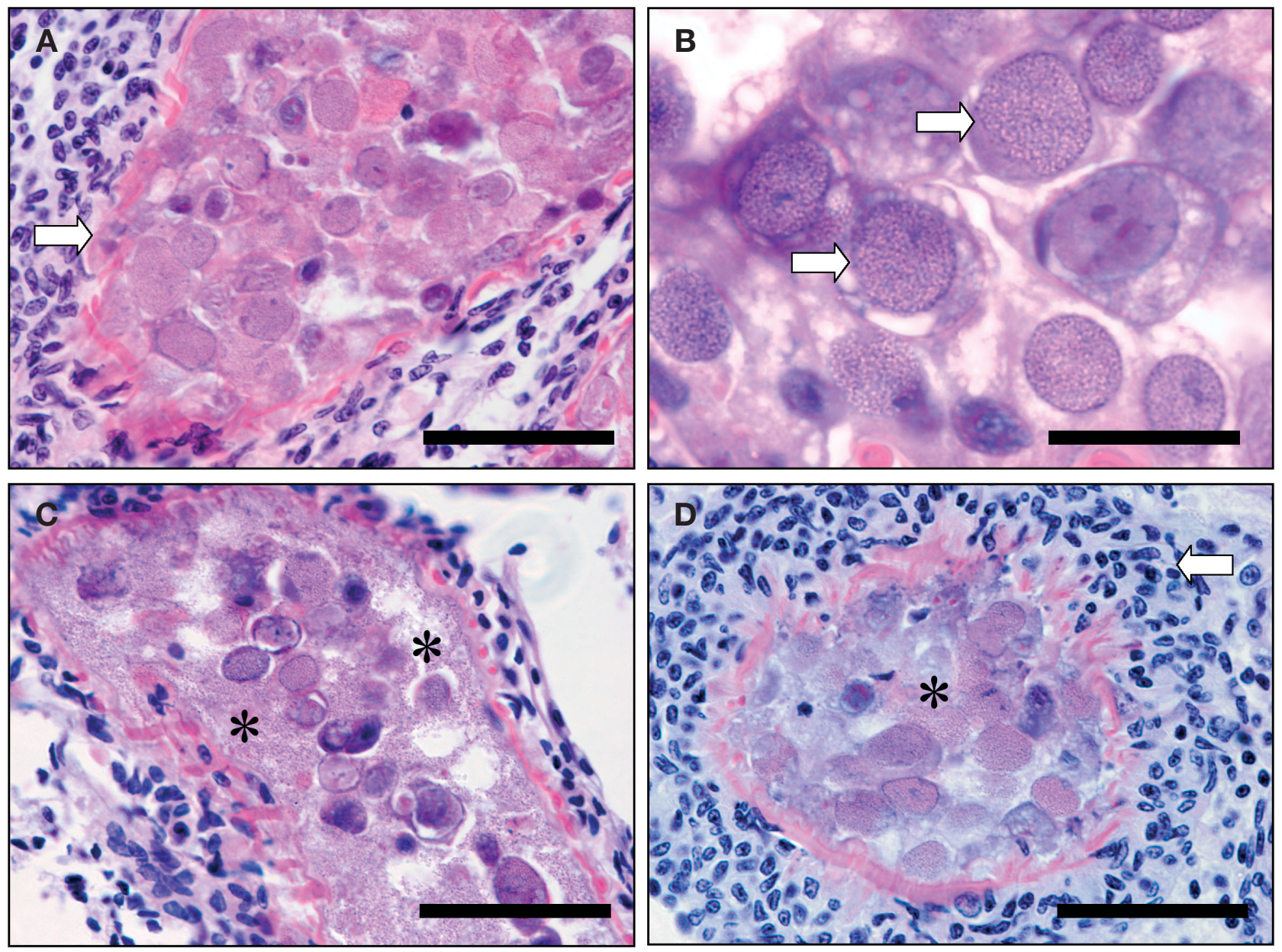

Fig. 1. Enterospora sp. infecting hepatopancreatic epithelia of Eupagurus bernhardus. Histopathology (A) heavily infected tubule showing dissociation of degenerate epithelial cells from existing basement membrane (arrows); sloughed infected cells fill tubule lumen. (B) Sloughed epithelial cells with hypertrophic, eosinophilic and granular nuclei (arrows); host chromatin is restricted to outer margins of infected nuclei. (C) Heavily infected tubule with sloughed epithelial cells and foci of bacterial microcolonies (asterisks). (D) Heavily infected tubule with disassociated epithelial cells, bacterial microcolonies (asterisk) and pronounced infiltration by host haemocytes (arrow). All images H\&E. Scale bars $=(A, C, D) 100 \mu m$, (B) $20 \mu \mathrm{m}$

tebrate and follows a description by our laboratory of a new genus of intranuclear microsporidians infecting decapod crustaceans (Stentiford et al. 2007). The parasite from hermit crabs was discovered during routine monitoring of commercial and sentinel species during the UK NMMP in June 2003. As such, we can make no inference as to the prevalence of infection in hermit crabs at different times of the year nor at different locations.

A key feature of microsporidians within the family Enterocytozoonidae is the formation of spore organelles (extrusion apparatus such as the polar filament and anchoring disk) prior to fission of the sporogonic plasmodium into uninucleate sporoblasts and spores. The parasite described herein exhibits this key developmental feature and, as such, resides within this fam- ily. Furthermore, due to the close similarity of this parasite with Enterospora canceri from Cancer pagurus (crustacean host, hepatopancreatic habit, intranuclear, organelles formed within plasmodium, 4 to 5 turns of polar filament, similar spore size, high spore production) it is appropriate that it is also placed within the same genus. Due to the low apparent prevalence of infection in the population sampled and the lack of opportunity to collect additional material from this population, it has not been possible to classify the parasite from the hermit crab to the species level. While key developmental stages clearly define the parasites from hermit crab and edible crab as genus relatives, the hermit crab parasite exhibited an intriguing feature that was not seen in the edible crab parasite (Stentiford et al. 2007). This feature involved the asso- 

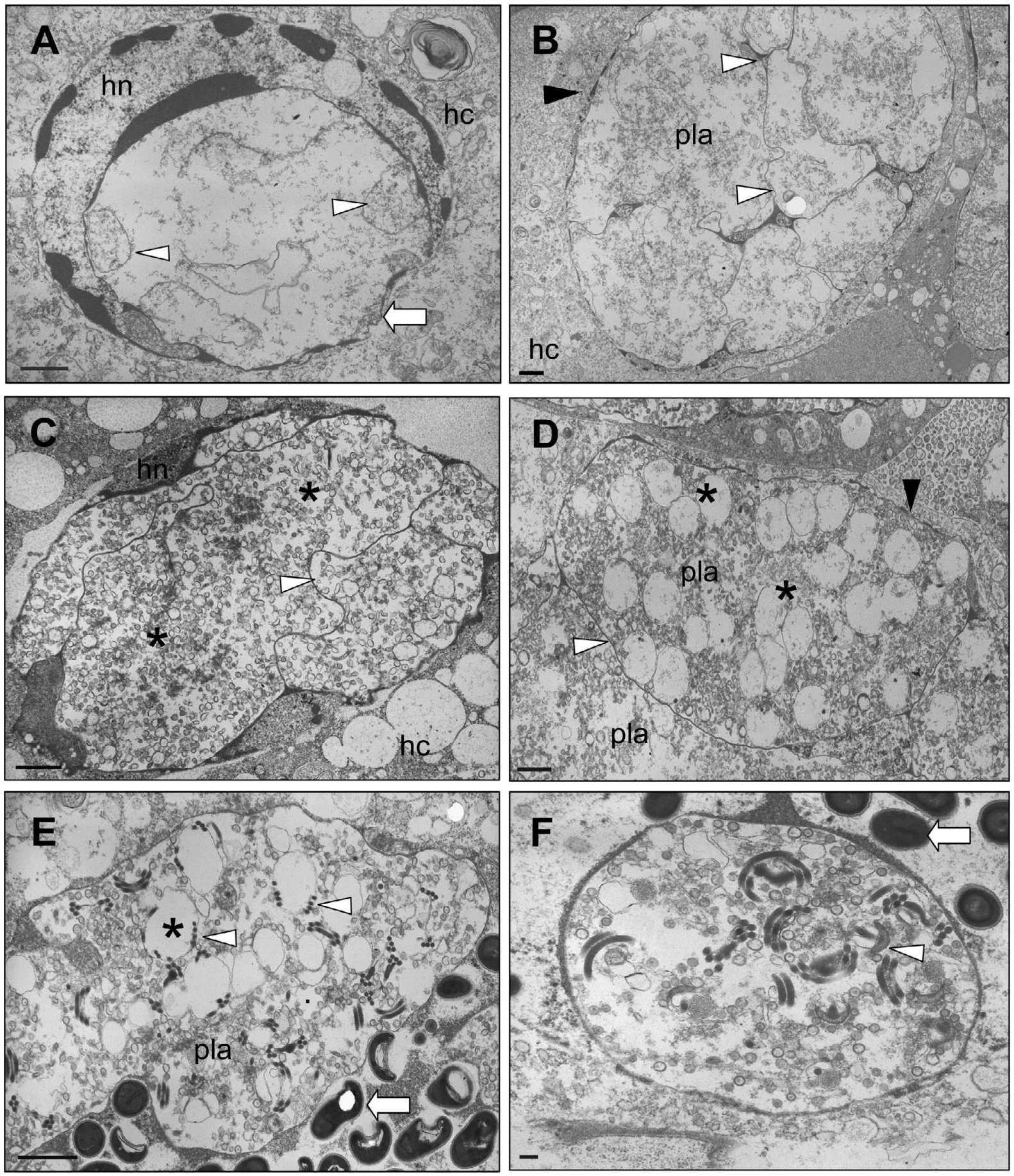

Fig. 2. Enterospora sp. infecting hepatopancreatic epithelia of Eupagurus bernhardus. Ultrastructure of early life stages. (A) Early meront stage (arrow) within nucleoplasm of host cell nucleus (hn); arrowheads: parasite nuclei; hc: host cell cytoplasm. (B) Early plasmodial stage filling nucleoplasm of host cell (black arrowhead); plasmodium (pla) contains internal septa (white arrowheads); hc: host cell cytoplasm. (C) Early plasmodial stage with internal septa (arrowhead) and small membrane-bound vesicles (asterisks); hn: host cell nuclei; hc: cytoplasm. (D) Early plasmodium (pla) with paired nuclei (asterisks) and internal septa (white arrowhead); different regions of plasmodium appear to be at different developmental stages; black arrowhead: host cell nuclear border. (E) Developing sporogonal plasmodium (pla) with large electron-lucent vacuoles (asterisk) and associated precursors of spore extrusion apparatus (white arrowheads); mature spores are visible outside plasmodium and in direct contact with host nucleoplasm (arrow). (F) Section of sporogonal plasmodium showing developing polar filaments with 4 to 5 coils and terminating in dome-shaped anchoring disk (arrowhead); mature spores are visible outside plasmodium in direct contact with host nucleoplasm (arrow). Scale bars $=(A$ to E) $1 \mu \mathrm{m},(F) 200 \mathrm{~nm}$ 

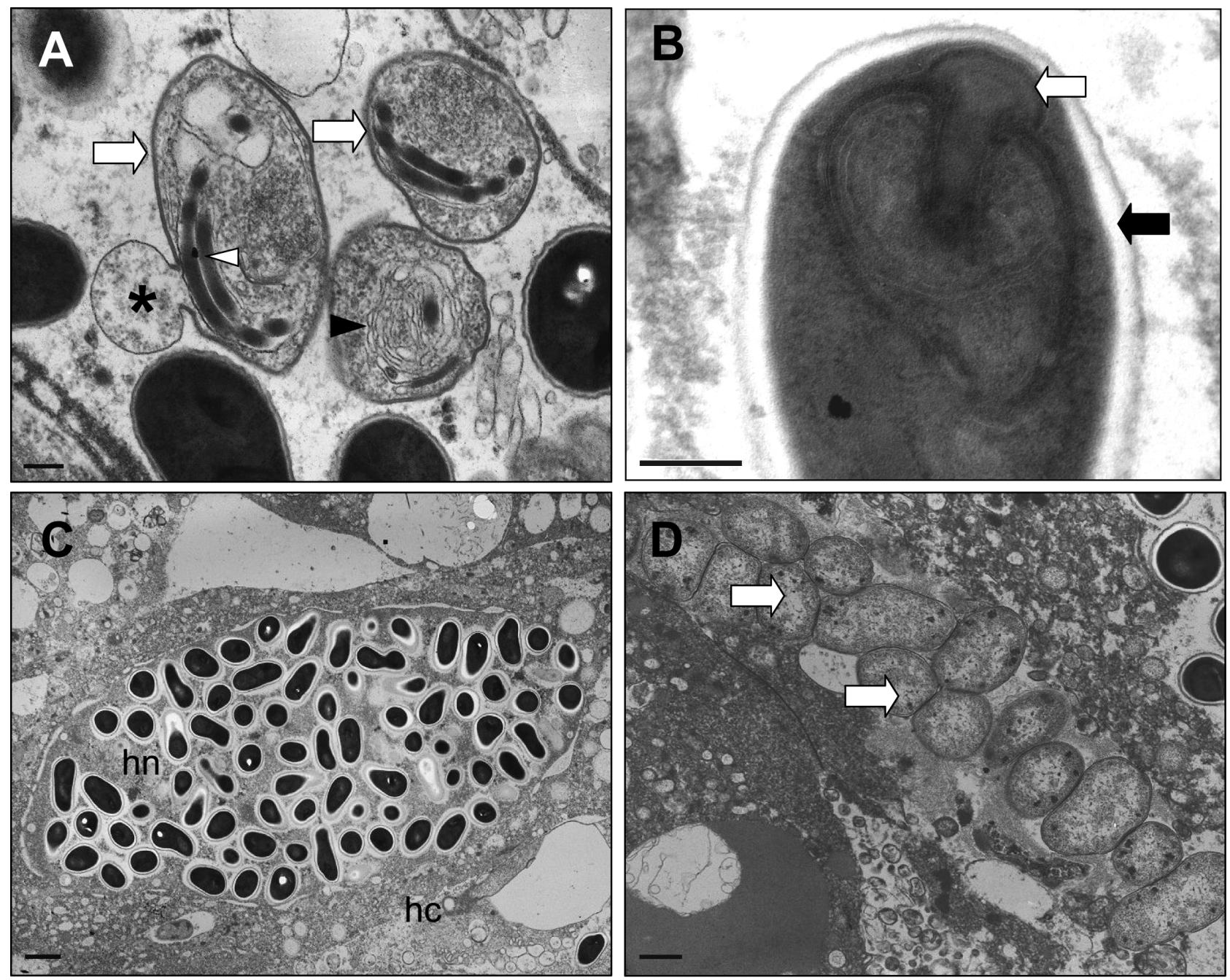

Fig. 3. Enterospora sp. infecting hepatopancreatic epithelia of Eupagurus bernhardus. Ultrastructure of spore development. (A) Sporoblast stages (arrows) with polar filament precursors (white arrowhead) and polaroplast (black arrowhead); small protrusions were occasionally observed in sporoblast wall (asterisk). (B) Maturing spore showing anchoring disk (white arrow) and trilaminate wall (black arrow). (C) Host nuclei during advanced infection; nucleoplasm (hn) is filled with mature spores; host cytoplasm (hc) is unaffected at this stage. (D) Late infection with microcolonies of bacteria (arrows) amongst liberated microsporidian spores and cell debris within tubule lumen. Scale bars $=(A, B) 200 \mathrm{~nm},(\mathrm{C}, \mathrm{D}) 1 \mu \mathrm{m}$

ciation of polar filament precursors with the large electron lucent vacuoles present within the plasmodial cytoplasm (Fig. 2E). Whether this feature is common amongst parasites of the genus Enterospora or perhaps a unique feature of the parasite from hermit crabs will require analysis of further specimens.

Recent studies of microsporidian taxonomy based upon analysis of regions of the parasite genome have placed members of the Enterocytozoonidae as basal members within the microsporidia (Lom \& Nielsen 2003). Since the crustacean parasites within the newly formed Enterospora appear to reside within the same family, it is feasible that a taxonomic comparison based upon genomic and ultrastructural features may now allow firmer links to be drawn between the disparate Enterocytozoonidae and all other microsporidian families. A recent molecular phylogenetic study of a microsporidian from crustacean sea lice revealed similarity with the Enterocytozoonidae (particularly the Nucleospora) (Freeman et al. 2003) and Refardt et al. (2002) report that the daphnid parasite Microsporidium sp. also appears closely related to the Enterocytozoon. To this end, further work should gather appropriate Enterospora-infected material from Cancer pagurus and Eupagurus bernhardus and attempts made to compare these and other hepatopancreatic microsporidians from crustaceans with those found within higher animals. 


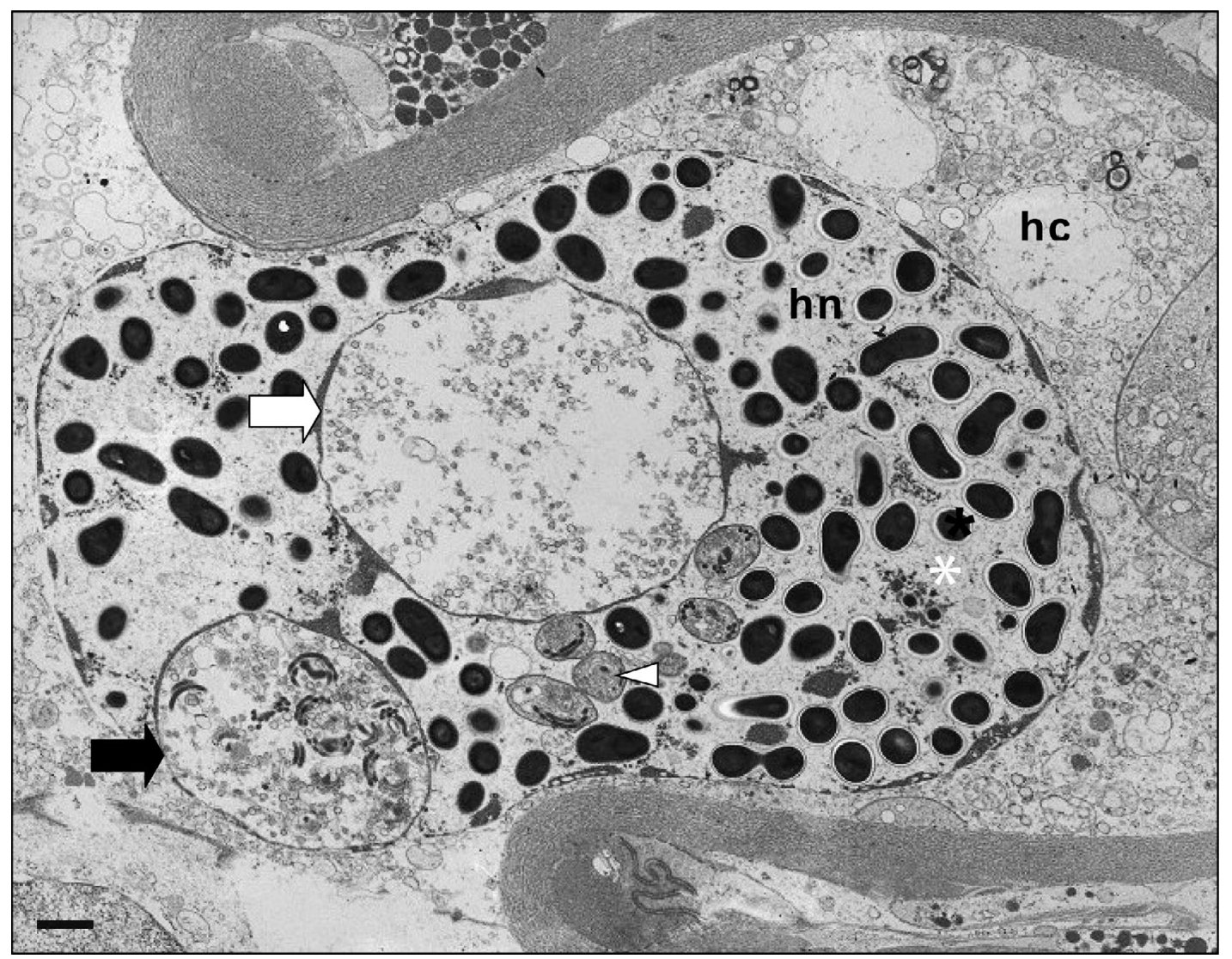

Fig. 4. Enterospora sp. infecting Eupagurus bernhardus. Ultrastructure of asynchronous development of Enterospora sp. within a single host nucleus (hn). Early meront (white arrow), sporogonal plasmodium (black arrow), liberated sporoblast (arrowhead) and mature spores (asterisk) are present. Host cytoplasm (hc) is unaffected at this stage. Scale bar $=1 \mu \mathrm{m}$

Acknowledgements. The authors thank the crew of the RV 'CEFAS Endeavour' for assistance with capture of specimens. This work was supported by the Department of Environment, Food and Rural Affairs (DEFRA) under contract numbers AE003 and F1166.

\section{LITERATURE CITED}

Freeman MA, Bell AS, Sommerville C (2003) A hyperparasitic microsporidian infecting the salmon louse, Lepeoptheirus salmonis: an rDNA-based molecular phylogenetic study. J Fish Dis 26:667-676

Hopwood D (1996) Fixation and fixatives. In: Bamcroft JD, Stevens A (eds) Theory and practice of histopathological

Editorial responsibility: Timothy Flegel, Bangkok, Thailand techniques, 4th edn. Churchill Livingstone, Hong Kong, p 23-46

Lom J, Nilsen F (2003) Fish microsporidia: fine structural diversity and phylogeny. Int J Parasitol 33:107-127

Refardt D, Canning EU, Mathis A, Cheney SA, LafranchiTristem NJ, Ebert D (2002) Small subunit ribosomal DNA phylogeny of microsporidia that infect Daphnia (Crustacea: Cladocera). Parasitology 124:381-389

Reynolds ES (1963) The use of lead citrate at high $\mathrm{pH}$ as an electron-opaque stain in electron microscopy. J Cell Biol 17:208-212

Stentiford GD, Bateman KS, Longshaw M, Feist SW (2007) Enterospora canceri n. gen., n. sp., intranuclear within hepatopancreatocytes of the European edible crab Cancer pagurus. Dis Aquat Org 75:61-72

Submitted: July 27, 2006; Accepted: February 2, 2007 Proofs received from author(s): March 22, 2007 